\title{
Impedance eduction of perforated plates at low Strouhal numbers and high bias flow Mach number
}

\author{
V. Popie, E. Piot $†$ F. Simon ${ }^{\ddagger}$ \\ ONERA - The French Aerospace Lab, F-31055, Toulouse, France \\ S. Tordeux ${ }^{\S}$ \\ INRIA Bordeaux - Sud-Ouest, Pau, France
}

\begin{abstract}
The knowledge of the acoustic behavior of a multiperforated plate is important to design a combustion chamber. It is characterized by the acoustic impedance or the Rayleigh conductivity. Experiments have been performed in the ONERA flow duct to study the acoustic response of a multiperforated plate, without and with bias flow. The geometry of the perforated plate is representative of industrial applications, as well as the bias flow Mach number (around 0.3) and the Strouhal number (around $5 \cdot 10^{-3}$ ). An inverse method is used to educe the impedance of the plate from Laser Doppler Anemometry measurements of the acoustic velocity above the plate. The issue of the accuracy of the educed value is specifically adressed. It is shown that the perforated plate is highly reflective with bias flow, as predicted by the models available in the literature. This is due to the combination of the very low Strouhal number and high bias flow Mach number. Consequently, on contrary to the case without flow, in the configuration with bias flow the eduction process shows one's limits and cannot give a very precise impedance value.
\end{abstract}

\section{Nomenclature}

$c_{0}$

$t$

$Z$

Sound speed

Frequency

Angular frequency, $=2 \pi f$

Plate thickness

Perforation radius imaginary number Objective function Wave number, $=\omega / c_{0}$ Stokes wave number

Rayleigh conductivity Cavity depth

Mach number through the holes

Strouhal number, $=\omega r /\left(c_{0} M_{b}\right)$

cavity Helmholtz number, $=\omega L / c_{0}$ mass flow rate within the cavity

time

Acoustic impedance of the perforated plate

$Z_{t} \quad$ Acoustic impedance of the duct exit

Greeks

*PhD Student, ONERA DMAE-ITAC, vincent.popie@onera.fr

$\dagger$ Research Scientist, ONERA DMAE-ITAC, estelle.piot@onera.fr

‡Research Scientist, ONERA DMAE-ITAC, frank.simon@onera.fr

$\S$ Associate Professor, INRIA MAGIQUE-3D, sebastien.tordeux@inria.fr 
$\gamma \quad$ Strouhal dependent function defined by Howe ${ }^{1}$

$\mu \quad$ Dynamic viscosity

$\nu \quad$ Kinematic viscosity

$\rho_{0} \quad$ Density

$\sigma \quad$ Porosity of the perforated plate

$\phi \quad$ State vector

$\omega \quad$ Angular frequency

$\Omega_{\text {obs }}$ Observation region, which coincides with the LDA measurement field

Subscript

DG Discontinuous Galerkin computed quantity

Meas Measured quantity

Acronyms

LDA Laser Doppler Anemometry

SPL Sound Pressure Level

\section{Introduction}

Perforated plates with bias flow are used in the combustion chamber for cooling purpose, but can also act as dampers with regards to acoustic waves. Characterizing their acoustic behavior is thus of primary interest in the framework of combustion chambers thermo-acoustic instabilities. This behavior is usually studied through the absorption coefficient, that is directly related to the Rayleigh conductivity (and the acoustic impedance) of the plate in the basic setup in which the plate is reflecting a normally incident acoustic wave within a tube. The Rayleigh conductivity depends on the perforated plate geometrical features (mainly the thickness of the plate, the size and shape of the perforations) and the environmental conditions (mean flow, sound pressure level).

In most experimental studies (see for instance ${ }^{2-4}$ ), the bias flow is low or the perforations diameter is large, which leads to a Strouhal number much higher than in actual aeroengine combustor liners. In the combustion chambers, these liners are used primarily for film cooling purpose; however, from an acoustic point of view, the behavior of perforated plates is different from the one of a solid wall and therefore needs to be quantified. In this work, we apply to a perforated plate representative of real combustion walls a method developed for educing the impedance of inlet nacelle liners. ${ }^{5,6}$ The perforated plate can be subjected to a bias flow up to Mach 0.3, which leads to Strouhal numbers in the range of $5.10^{-3}$ (which is typical from industrial configurations ${ }^{7-9}$ ). These values are ten times smaller and the Mach number value is ten times higher, than the values studied by Scarpato et. al ${ }^{10,11}$ or Cosi et. al ${ }^{12}$ for designing Helmholtz acoustic dampers (implemented within the combustion chamber for mitigating the thermo-acoustic instabilities).

The plate is placed at the wall of the ONERA B2A bench, above a cavity whose depth is chosen so that the associated Helmholtz number is of order unity. Acoustic waves are travelling parallel to the plate, which means that, on contrary to most of the previous experimental or numerical studies, ${ }^{2,10,13}$ we consider grazing rather than normal incident pressure waves. Perforations are orthogonal to the plate, and their geometry (size, porosity) is representative of generic perforated plates used in combustion chamber. The acoustic source is a multi-sine signal. In the case without flow, the source sound pressure level is around $115 \mathrm{~dB}$, in order to stay in the linear regime of the plate impedance, while with bias flow it is around $140 \mathrm{~dB}$. The impedance of the plate is educed from the measurements of the acoustic velocity within the vein, performed through laser velocimetry.

First, the ONERA B2A bench and the characteristics of the perforated plate are presented. Then, a review on models existing in the literature for the Rayleigh conductivity and the acoustic impedance of the plate is done. Finally, the eduction method is presented and results with and without bias flow are compared with existing models. 


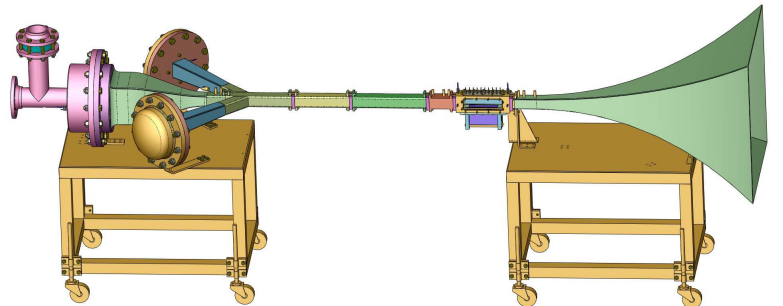

ᄂ

(a) Global view of B2A duct

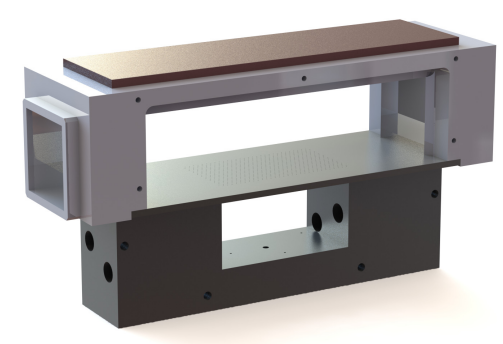

(b) Zoom on the testing cell, which shows the perforated plate above the cavity, and the lateral holes for flow injection.

Figure 1. Sketch of B2A Aero-Thermo-Acoustic duct.

\section{Measurements}

\section{A. ONERA B2A duct}

The aeroacoustic test bench at Onera (B2A) is made of a stainless tube of section $50 \mathrm{~mm} \times 50 \mathrm{~mm}$; its total length is about $4 \mathrm{~m}$. A $200 \mathrm{~mm}$-long test section is equipped with two silica windows for optical access. In the present experiments, a perforated plate is put at the bottom of the test section, over a cavity (see Fig. 1).

The perforated plate spans the whole width of the duct: it is a $150 \mathrm{~mm} \times 50 \mathrm{~mm}$ rectangle. The perforations are untilted cylinders of diameter $d=0.45 \mathrm{~mm}$. The thickness of the plate is $h=4 \mathrm{~mm}$. It was chosen so that it matches the effective thickness of a realistic plate perforation, which is tilted with a $60^{\circ}$ angle. The perforations are centered on the plate on a $90 \mathrm{~mm} \times 48.6 \mathrm{~mm}$ zone, which yields to a plate porosity of $\sigma=1.96 \%$. The cavity is $L=50 \mathrm{~mm}$ deep, $50 \mathrm{~mm}$ wide and $90 \mathrm{~mm}$ long : it is located exactly below the perforated section. The cavity depth was chosen so that the Helmholtz number is of order unity. The cavity can be fed by a flow through four lateral holes drilled in its vertical walls, in a way similar to the one used in a previous study. ${ }^{14,15}$ The flow rate can be controlled to produce a bias flow through the plate. It must be noticed that for all the experiments shown in this paper, there is no grazing flow within the duct.

Two acoustic drivers are mounted upstream of the test section, and are used to generate tones (multi-sine signal) at up to $140 \mathrm{~dB}$ over a frequency range of 260 to $620 \mathrm{~Hz}$, that is well below the cut-off frequency of the duct (which is $3.5 \mathrm{kHz}$ ). The termination is equipped with a quasi-anechoic outlet, leading to an upstream reflection coefficient smaller than 0.2 for frequencies higher than $350 \mathrm{~Hz}$,as shown in Fig. 2). A 2C fringe mode LDA-system is used to measure the axial (x-coordinate) and vertical (z-coordinate) components of the acoustic velocity, for each frequency of the multi-sine source signal. Details on the measurement technique and on the post-treatment used for obtaining the acoustic component from the LDA velocity may be found in previous papers. ${ }^{6,15,16}$ The measurement domain is a $150 \mathrm{~mm}$ long and $30 \mathrm{~mm}$ high rectangle, located at mid-span of the duct and just above the bottom wall of the duct. It is meshed with 300 measurements points. It extends $30 \mathrm{~mm}$ upstream of the start of the perforated zone and $30 \mathrm{~mm}$ downstream of its end. A sketch of the measurement configuration is shown in Fig. 3.

\section{B. Experimental results}

The experimental campaign was carried out in two steps. First, the acoustic response of the plate was studied without bias flow, and with a SPL at each tone frequency around $115 \mathrm{~dB}$. This low value of the sound source ensures that the perforated plate impedance stays in the linear regime. An example of the LDA measurements is plotted in Fig. 4. It shows the amplitude of $u_{z}$, at $f=500 \mathrm{~Hz}$, in almost all the $\Omega_{o b s}$ region (only the last $x$ and $z$ lines of the mesh are omitted). The amplitude is small (a few $\mathrm{mm} / \mathrm{s}$ ), but not zero at it would be if the plate was perfectly rigid. 


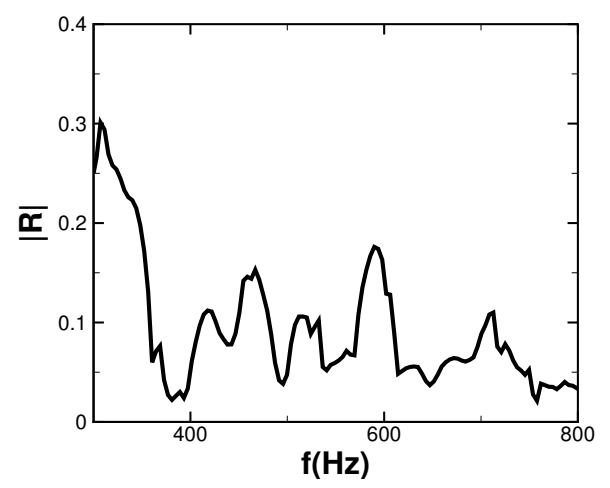

Figure 2. Reflexion coefficient of the B2A termination, in the studied range of frequency.

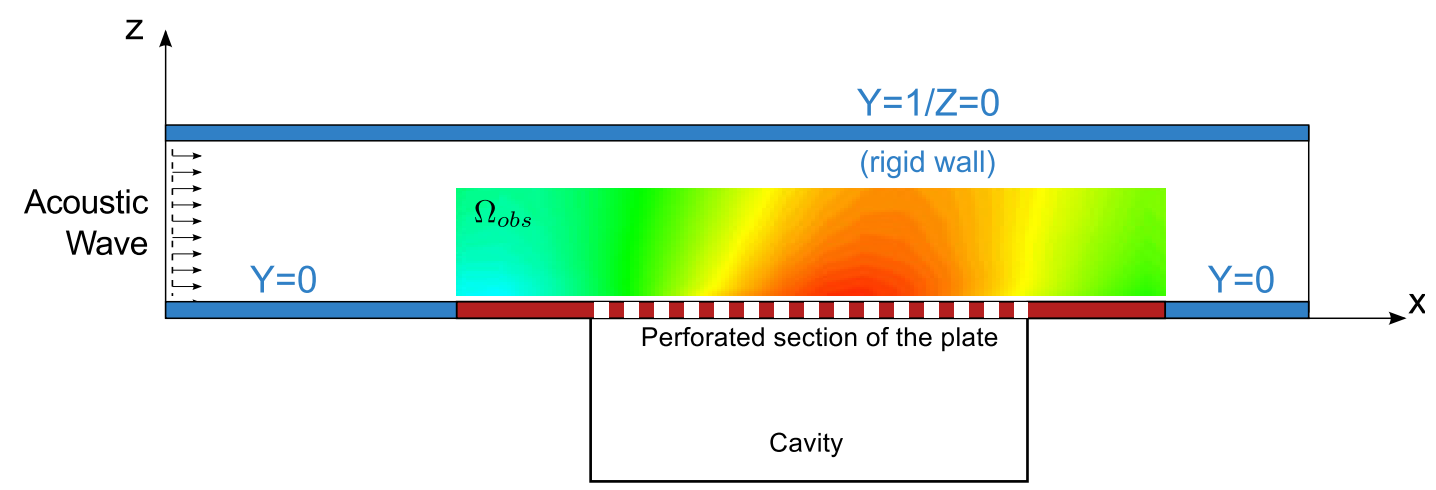

Figure 3. Sketch of the experimental configuration, with the LDA measurement region denoted as $\Omega_{o b s}$. The perforated plate is drawn in red, the perforated zone is shown with white stripes.

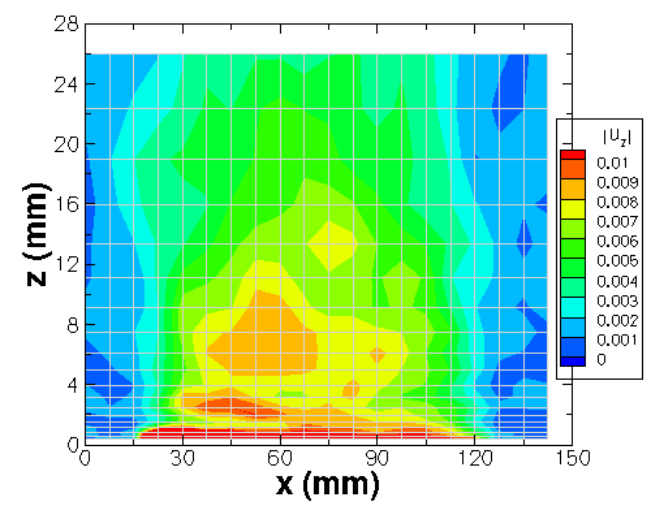

Figure 4. Amplitude (in $\mathrm{m} / \mathrm{s}$ ) of the vertical component of the acoustic velocity field, at $f=500 \mathrm{~Hz}$. Configuration without any mean flow. The perforated zone is located at $z=0$ and between $x=30$ and $x=120$ mm. 


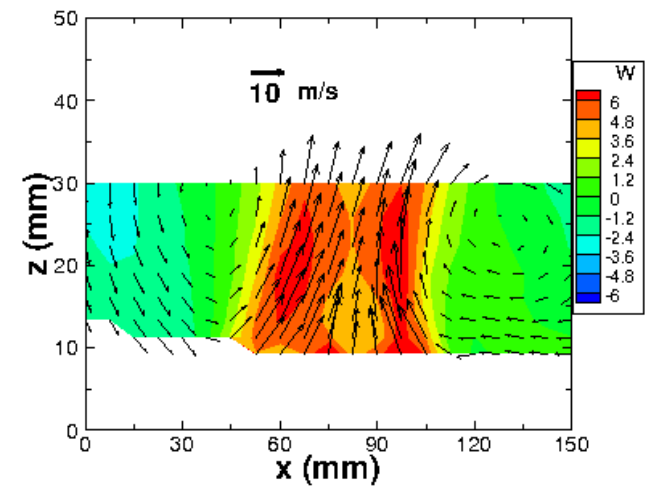

(a) Velocity field of the bias flow. The colored contours show the vertical mean velocity, in $\mathrm{m} / \mathrm{s}$

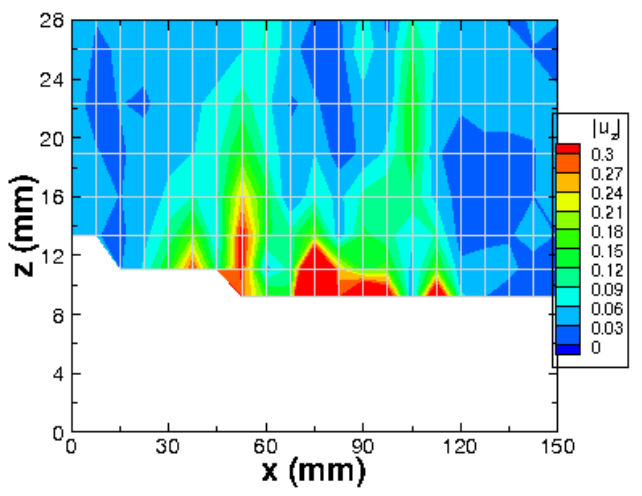

(b) Amplitude (in $\mathrm{m} / \mathrm{s}$ ) of the vertical component of the acoustic velocity field, at $f=500 \mathrm{~Hz}$.

Figure 5. LDA measurements in the configuration with a $M_{b}=0.3$ bias flow. The perforated zone is located at $z=0$ and between $x=30$ and $x=120 \mathrm{~mm}$.

Then, the acoustic response of the plate was studied with bias flow. Configurations with a Mach number through the holes equal to 0.1, 0.2 and 0.3 have been tested. The Mach number value is estimated from the mass flow rate injected within the cavity, and under the assumption of a discharge coefficient $C_{D}=0.62$ for the holes (quasi-steady limit, see ${ }^{17}$ ) :

$$
q=\rho_{0} c_{0} C_{D} M_{b} \sigma S
$$

where $S$ is the surface of the perforated zone ${ }^{\text {a }}$. LDA measurements have only been performed for the $M_{b}=0.3$ configuration, i.e. for Strouhal numbers in the range of $\left[3.10^{-3}, 8.10^{-3}\right]$. This range of Strouhal numbers is two order of magnitude less than that studied by Scarpato et. al. ${ }^{10}$ To ensure a correct measurement of the acoustic velocity field, the SPL at each tone frequency was around $140 \mathrm{~dB}$. It is expected that, in such a bias flow configuration, the acoustic response of the plate is driven by the bias flow rather than by the source level, and therefore the plate impedance should stay in the linear regime with respect to the SPL value. An example of the LDA measurements is plotted in Fig. 5. The mean flow is shown in Fig. 5(a), the vertical acoustic velocity in Fig. 5(b). Because of seeding issues, the LDA measurements give reliable results above $z=9 \mathrm{~mm}$ only. However, the measurement region is large enough to observe a non-zero vertical acoustic velocity above the perforated zone.

\section{Acoustic impedance models}

The acoustic response of the perforated plate is assessed via its acoustic impedance or the Rayleigh conductivity of the perforations. In this paper, these values are given in the frequency domain, following the $e^{\mathrm{j} \omega t}$ convention. Existing models can be classified by physical effects taken into account. We will consider only two of them.

$\mathrm{Maa}^{20}$ proposed a model based on Crandall's, ${ }^{21}$ Ingard's ${ }^{22}$ and Melling's $\mathrm{s}^{23}$ results for acoustic impedance of a perforated plate without mean flow :

$$
\rho_{0} c_{0} Z_{p}=\frac{8 \mu}{r^{2}} h \sqrt{1+\frac{r^{2} k_{s}^{2}}{32}}+\frac{1}{2} \sqrt{2 \omega \rho_{0} \mu}+\mathrm{j} \omega \rho_{0}\left[h\left(1+\left(3^{2}+\frac{r^{2} k_{s}^{2}}{2}\right)^{-\frac{1}{2}}\right)+\frac{16 r}{3 \pi}\right]
$$

where $k_{s}=\sqrt{\frac{\omega \rho_{0}}{\mu}}$. This model takes into account the acoustic energy dissipation via viscous friction at the walls of the holes. Only the linear response of the plate with respect to the source sound pressure level is considered.

\footnotetext{
a it must be emphasized that the choice of the discharge coefficient value may be questionable. Indeed, the plate perforations are rather long $(h /(2 r) \approx 9)$ and the Reynolds number may be low enough so that the jet flow is laminar. In such a case, a higher value of the discharge coefficient may be expected, around $0.72 .^{18,19}$ However, this value is highly dependent on the rounding of the orifice egdes, and a dedicated study would be necessary.
} 
When there is a bias flow through the holes, the mechanism of acoustic energy dissipation is fundamentally different from the one modelled by Maa. Acoustic energy is converted into vortical energy within the mean jet exiting the hole, and then the vortical energy is dissipated by heat and viscosity. Howe ${ }^{1}$ proposed a model for these mechanisms. It characterizes the Rayleigh conductivity of a hole in a thin plate under bias flow, and reads :

$$
K_{R}=2 r(\gamma+\mathrm{j} \delta)
$$

where $\gamma$ and $\delta$ depends on the Strouhal number and are defined in Howe's paper ${ }^{\mathrm{b}}$. According to Howe, the reference velocity used in the Strouhal number definition is the convection velocity of the vortex created at the aperture edges and swept away by the jet flow. Howe mentioned that this velocity equals half that of the jet velocity in the contracted section. However, in most of the studies, ${ }^{4,11,24}$ the reference velocity in the Strouhal number is defined as the mean bias flow velocity in the hole. Indeed, the correct value of the scaling factor depends on the detailed geometry of the orifice and the flow in this region. In this paper, we will follow this convention and define the Strouhal number with respect to $M_{b}$. The scaling factor is taken into account through the value of the discharge coefficient used for calculating $M_{b}$ from the flow rate in Eq. (1).

Howe's model has been adapted for thick plates ${ }^{25}$ :

$$
K_{R}=2 r\left(\frac{1}{\gamma+\mathrm{j} \delta}+\frac{2}{\pi} \frac{h}{r}\right)^{-1}
$$

The plate impedance associated to the Rayleigh conductivity is given by the relation :

$$
\rho_{0} c_{0} Z_{p}=\frac{j \omega \rho_{0} \pi r^{2}}{\sigma K_{R}}
$$

In the experiments, the perforated plate is mounted above a cavity, whose impedance is only imaginary and is equal to:

$$
\rho_{0} c_{0} Z_{c}=-\mathrm{j} \rho_{0} c_{0} \cot (k L)
$$

It must be noticed that this expression is only true when the cavity backplate is fully rigid and when the acoustic waves within the cavity propagate in a medium at rest. These hypotheses are verified in both studied configurations, since the mean flow in the cavity is negligible even in the bias flow case.

Finally, the specific impedance of the plate+cavity system is given by :

$$
Z=Z_{p}+Z_{c}
$$

A comparaison between the $Z$ value obtained without and with bias flow (as given by Maa's and Howe's models, respectively) is displayed in figure 6 . We can see that the resonance frequency of the system (associated to a reactance equals to zero) is close to $500 \mathrm{~Hz}$, and does not change significantly with or without bias flow. This is due to the choice of the cavity Helmholtz number and to the small influence of the perforated plate reactance on the reactance of the global system. However, the resistance changes dramatically when there is a bias flow. Indeed, for the very low Strouhal numbers considered in this paper, the Rayleigh conductivity tends to zero and the acoustic impedance to the infinity : the plate is very resistive and its behavior is close to a rigid wall. This is not the case without bias flow: Maa's model gives a resistance close to 1.5. This change of behavior with bias flow can be illustrated by looking at the reflection and absorption coefficients that would be obtained if the 'perforated plate+cavity' was submitted to sound pressure plane waves with a $\theta$ incidence angle (normal incidence, as obtained for instance in an impedance tube, corresponds to $\left.\theta=0^{\circ}\right)$. In this case, the reflection coefficient is defined as $R=(Z \cos \theta-1) /(Z \cos \theta+1)$ and the absorption coefficient as $\alpha=1-|R|^{2}$. When $\theta=0^{\circ}$, the maximum of absorption is obtained when the impedance is equal to $1+0 j$. It is important to notice that this 'optimal' value of the impedance is limited to the normal incidence configuration with acoustic plane waves: when the 'perforated plate+cavity' system is placed at the wall of a duct, the optimal impedance may be quite different; it depends on the duct geometry, the modal content of the source and the incidence of the sound waves (see Tester's papers for a discussion on this issue $\left.{ }^{26,27}\right)$. On Fig. 7 , results are plotted for both the normal incidence $\left(\theta=0^{\circ}\right)$ and a close-to-grazing incidence $\left(\theta=70^{\circ}\right)$, for illustration; indeed, the true angle of incidence obtained in

\footnotetext{
${ }^{\mathrm{b}} \gamma+\mathrm{j} \delta$ is used rather than $\gamma-\mathrm{j} \delta$ as in Howe's paper, because we use the $e^{\mathrm{j} \omega t}$ convention rather than the $e^{-\mathrm{j} \omega t}$ ansatz.
} 


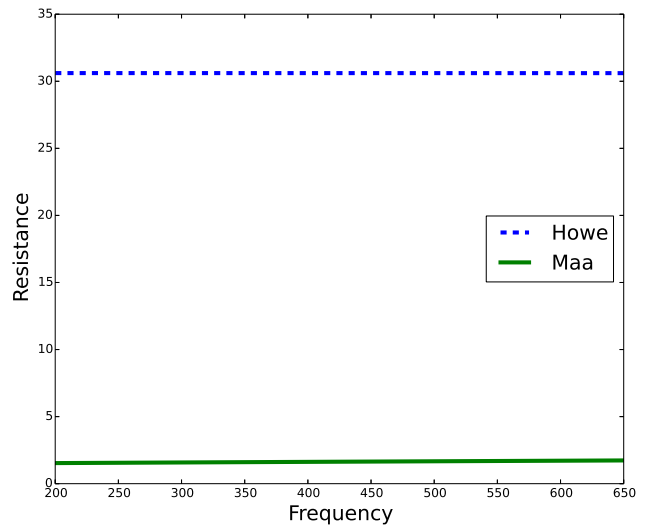

(a) Real part of $Z$

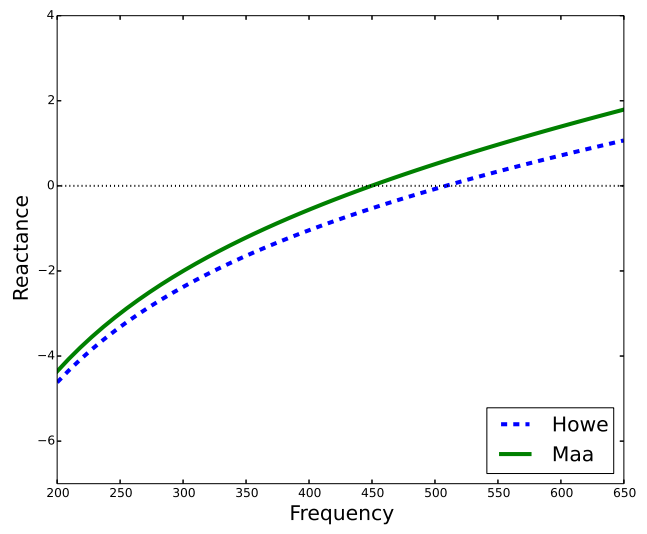

(b) Imaginary part of $Z$

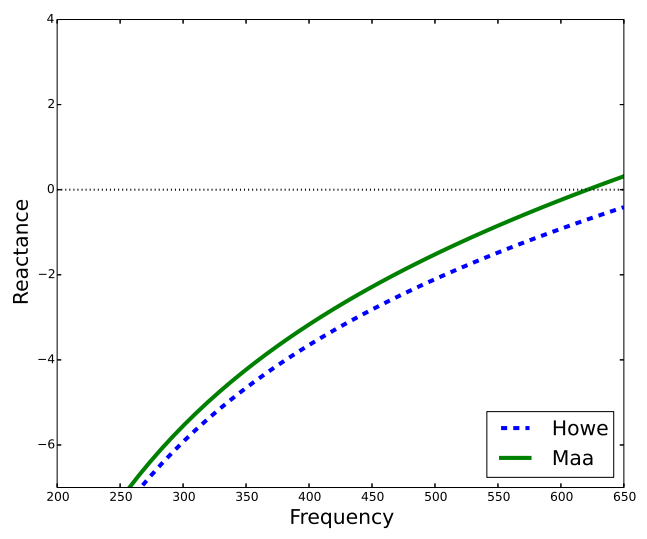

(c) Imaginary part of $Z_{p}$

Figure 6. Resistance (a) and reactance (b) of the system 'perforated plate+cavity' given by Howe's model (i.e. with bias flow $M_{b}=0.3$ ) and Maa's model (i.e. without flow). Subfigure (c) shows the reactance of the perforated plate only. 


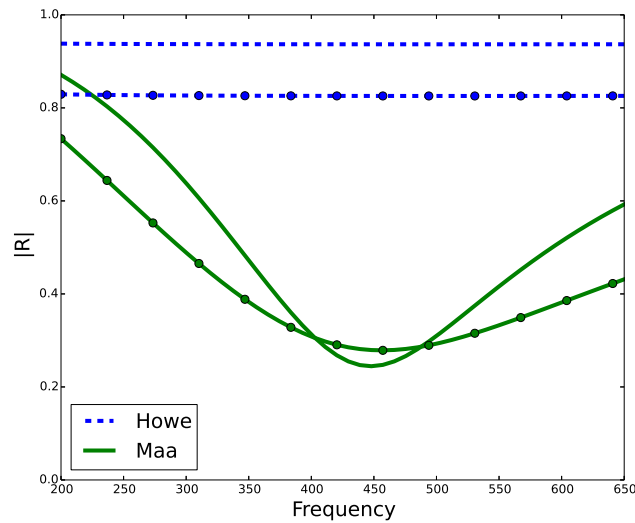

(a) Amplitude of the reflection coefficient

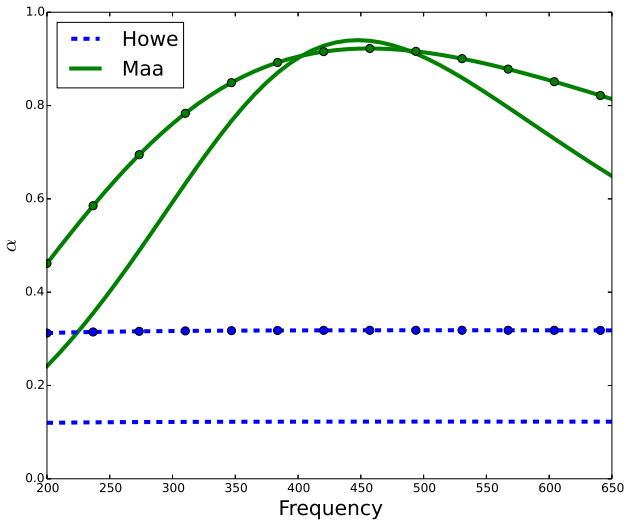

(b) Absorption coefficient

Figure 7. Reflexion and absorption coefficients associated to the impedance of the 'perforated plate+cavity' system submitted to normal incidence plane waves (lines without symbols) and waves with a $70^{\circ}$ incidence (lines with symbols). The impedance with a $M_{b}=0.3$ bias flow is given by Howe's model, the impedance without flow by Maa's model.

the tested experimental configuration is not known but we can infer that it is between 45 and $90^{\circ}$. Figure 7(b) shows that, with bias flow, the absorption at close-to-grazing incidence is higher than that at normal incidence, however the absorption remains much lower than without flow. We will see in newt section that it will make the impedance eduction difficult.

Finally, Fig. 7(a) confirms that at these very low Strouhal numbers, the perforated plate with a bias flow is almost fully reflective. This will stay true whatever the choice of the cavity depth (i.e. of the Helmholtz number), since this high reflection is due to the high value of the plate resistance. This behavior was already observed in numerical simulations of real industrial combustion chambers. ${ }^{28,}{ }^{29}$ It differs from the literature results showing that high absorption of perforated plates occurs for small values of the Strouhal number $S t \ll 1$. Indeed, as explained by Scarpato et. al, ${ }^{10,11}$ this statement is only true when $M_{b} \sim \sigma$, which is not the case in the present configuration: here $M_{b} \gg \sigma$.

\section{Impedance eduction}

\section{A. Methodology}

The impedance eduction method is based on the two-dimensional harmonic linearized Euler equations. The underlying model is briefly summarized here for convenience, but all details can be found in a previous paper. ${ }^{6}$ A discontinuous Galerkin scheme is chosen to solve the equations, in the domain drawn in Fig. 3 (only within the duct, the cavity is not included in the simulations). The numerical solution is denoted $\left.\phi\right|_{\text {DG }}$ whereas the measurements are denoted $\left.\phi\right|_{\text {Meas }}$. At the source plane, the state vector $\phi$ is taken to be equal to $\mathcal{C} \phi_{0}$, where $\phi_{0}$ is the plane-wave solution in a rigid duct without flow. As this plane-wave solution is chosen with an arbitrary amplitude taken to be equal to 1 , the complex coefficient $\mathcal{C}$ stands for the source amplitude. At the exit plane, either a non-reflecting or an exit impedance boundary condition can be enforced. The second option is used in this work, since it is known that the acoustic termination of the duct is not perfectly anechoic in the considered range of frequency. On the walls, an impedance boundary condition is enforced. To avoid any singularities on the rigid parts of the wall (drawn in blue on Fig. 3), the boundary condition is expressed as a function of the normal incidence reflection coefficient. The searched impedance value, $Z$, is imposed on the wall representative of the perforated zone, i.e. on the wall drawn with white stripes in Fig. 3. This impedance is therfore representative of the whole 'perforated plate+cavity' system .

The inverse problem is defined by the minimization of the following objective function :

$$
\mathcal{J}=\int_{\Omega_{\text {obs }}}\left\{\left.\phi\right|_{\mathrm{DG}}-\left.\phi\right|_{\text {Meas }}\right\}^{T}\left\{\left.\bar{\phi}\right|_{\mathrm{DG}}-\left.\bar{\phi}\right|_{\text {Meas }}\right\} \mathrm{dxdy}
$$


where $\Omega_{\mathrm{obs}}$ is the observation region and $\bar{\phi}$ is the complex conjugate of $\phi$. As only the velocity components are measured, the state vector $\phi$ is restricted to $\left(u_{x}, u_{z}\right)$ in the definition of the objective function.

A gradient algorithm is used to solve the optimization problem. The analytical expression of the gradient of $\mathcal{J}$ with respect to each of the search parameters $Z, Z_{t}, \mathcal{C}$ is obtained via an adjoint method, as explained in Primus et. al. ${ }^{5,6}$

The value of the educed impedance is obtained when the objective function $\mathcal{J}$ reaches its minimum. To assess the precision of the eduction process, we will consider a reduced value of the objective function, defined by :

$$
\mathcal{J}_{\text {red }}=\frac{\int_{\Omega_{\text {obs }}}\left\{\left.\phi\right|_{\text {DG }}-\left.\phi\right|_{\text {Meas }}\right\}^{T}\left\{\left.\bar{\phi}\right|_{\text {DG }}-\left.\bar{\phi}\right|_{\text {Meas }}\right\} \text { dxdy }}{\int_{\Omega_{\text {obs }}}\left\{\left.\phi\right|_{\text {Meas }}\right\}^{T}\left\{\left.\bar{\phi}\right|_{\text {Meas }}\right\} \text { dxdy }}
$$

which reaches its minimum value $\mathcal{J}_{\text {red }}^{\min }$ for the educed values of the impedance, exit impedance and source amplitude. This minimum value is typically around $0.3 \%$ : it differs from zero because of the inevitable measurements and numerical errors. The knowledge of the function shape around $\mathcal{J}_{\text {red }}^{\min }$ is important to analyze the results. The larger the variations of the function around the solution, the stronger the acoustical field depends on the value of the searched parameters. To characterize this effect, the reduced objective function can be displayed in the impedance plane, with $Z_{t}$ and $\mathcal{C}$ being kept at their optimal values. A range of resistance and reactance values in which $\left|\mathcal{J}_{\text {red }}-\mathcal{J}_{\text {red }}^{\min }\right|<0.1 \%$ can then be obtained: this can be understood as an uncertainty region on the educed impedance.

\section{B. Results without flow}

The eduction procedure has first been applied to the LDA measurements without bias flow. The educed impedance is plotted on Fig. 8 and compared to the impedance value $Z$ derived from Maa's model (Eq. (2)) and Eq. (6). A good agreement is obtained, which validates the impedance eduction methodology. At the lowest frequency, the large uncertainty value is due to the high value of the exit reflection coefficient.
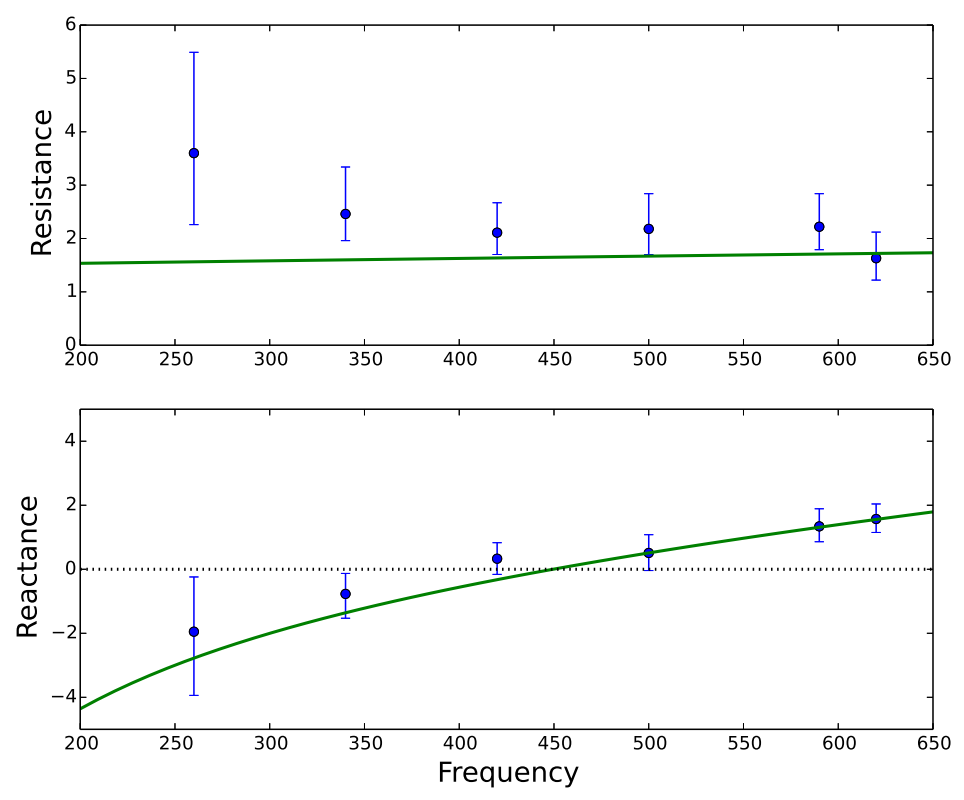

Figure 8. Educed impedance without bias flow (blue dots), compared to Maa's model (green solid line). Blue error bars display the range of resistance and reactance in which $\left|\mathcal{J}_{\text {red }}-\mathcal{J}_{\text {red }}^{\text {min }}\right|<0.1 \%$

An example of the reduced objective function map in the impedance plane is plotted in Fig. 9, for $f=500 \mathrm{~Hz}$. Here $\mathcal{J}_{\text {red }}^{\min }$ equals $0.17 \%$; the error bar displayed in Fig. 8 at this frequency corresponds therefore to the range of impedance in which $\mathcal{J}_{\text {red }}<0.27 \%$. 


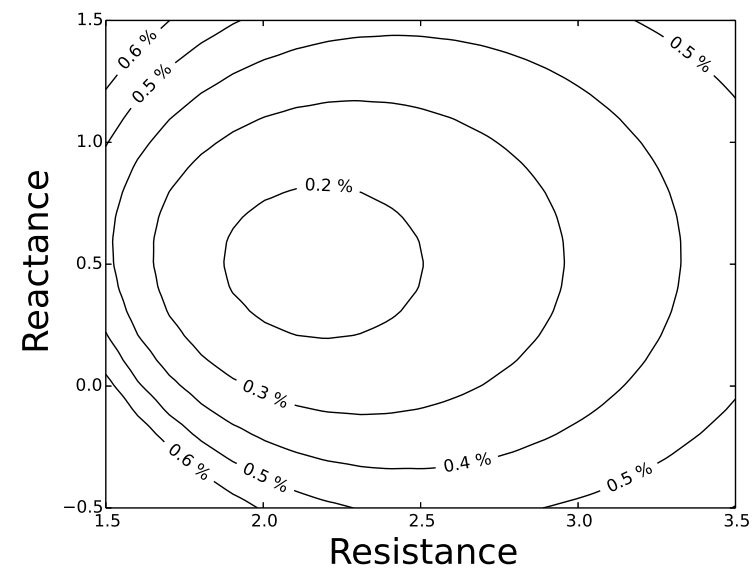

Figure 9. Map of the reduced objective function at $500 \mathrm{~Hz}$, in the impedance plane. The exit impedance and the source amplitude are fixed at their optimal values. Configuration without flow.

\section{Results with bias flow}

As expected from Howe's model, the perforated plate with a $M_{b}=0.3$ bias flow is highly resistive. Consequently, the acoustic absorption within the B2A duct is very low, and the aocustic velocity field measured by LDA is almost that of plane pressure waves traveling along a rigid wall. For this reason, the eduction procedure does not yield as precise impedance values as without flow. This will be illustrated at $f=500 \mathrm{~Hz}$ only, but holds whatever the frequency. At this frequency, Howe's model combined with Eq. (6) yields to an impedance $Z_{\text {Howe }}=30.6-0.07 j$. However, the eduction process converges to $Z_{\text {opt }}=43.6-13.5 j$, with a minimum value of $\mathcal{J}_{\text {red }}$ equal to $0.54 \%$. This large difference can be explained when looking at the shape of the reduced objective function, both in the source amplitude and impedance planes, as plotted in Fig. 10. In the source amplitude plane (Fig. 10(a)), the reduced objective function is convex, with steep gradients: a small variation of the source amplitude yields a significant change of the reduced objectif function level. However, the reduced objective function is very flat in the impedance plane (Fig. 10(b)): changing the impedance value from $Z_{\text {opt }}$ to $Z_{\text {Howe }}$ only increase $\mathcal{J}_{\text {red }}$ from $0.54 \%$ to $0.552 \%$. Such low levels of gradients do not allow a precise eduction of the impedance : the eduction procedure is too sensitive to possible local optima in the search parameters set. On a physical point of view, this means that the perforated plate is so resistive that even a large change on its impedance does not affect significantly the acoustic field within the duct. The main driver of the acoustic field is here the source amplitude (modulus and phase). It must be noticed that we would certainly have obtained more accurate results if the observation domain $\Omega_{o b s}$ would have been larger and closer to the wall (as realized in the experiments without flow). A new set of experiments in which the seeding issues would be resolved would therefore be beneficial.

\section{Conclusion}

The knowledge of the acoustic behavior of a multiperforated plate is important to design a combustion chamber; it is characterized by the acoustic impedance. Experiments were performed in the ONERA flow duct on a perforated plate representative of real combustion walls in configurations with and without bias flow. The plate was placed above a cavity designed to yield Helmholtz number in the range of unity. It was submitted to plane pressure waves in a grazing incidence, and the acoustic field within the duct was measured by LDA. The configuration with bias flow was chosen so that very low Strouhal numbers $\left(\sim 10^{-3}\right)$ and high bias flow Mach numbers $(\sim 0.3)$ were reached, in order to mimic the conditions encoutered by linings used for film cooling purpose at the walls of combustion chambers. The bias flow Mach number is here much higher than the perforated plate porosity, i.e. far from the optimal bias flow value. Consequently, the 'perforated plate above cavity' system shows an acoustic behavior quite different from the acoustic dampers previously studied in the literature: its acoustic resistance is very high, and therefore, even near the resonance frequency, 


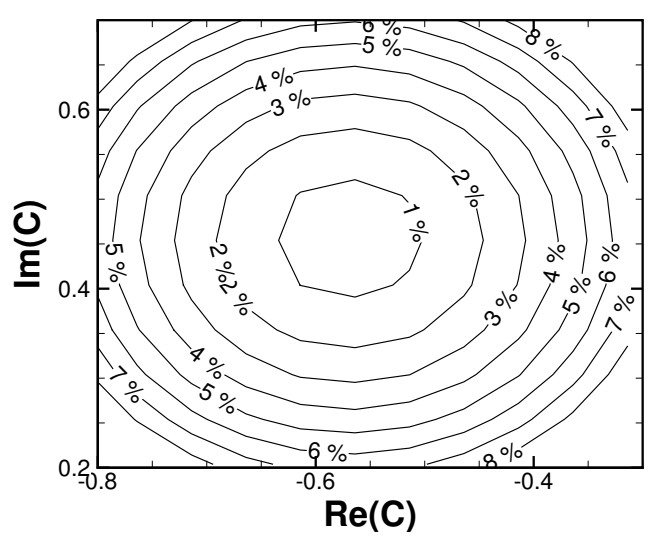

(a) Map in the source amplitude plane, when the impedance is fixed at $Z=Z_{\text {Howe }}$.

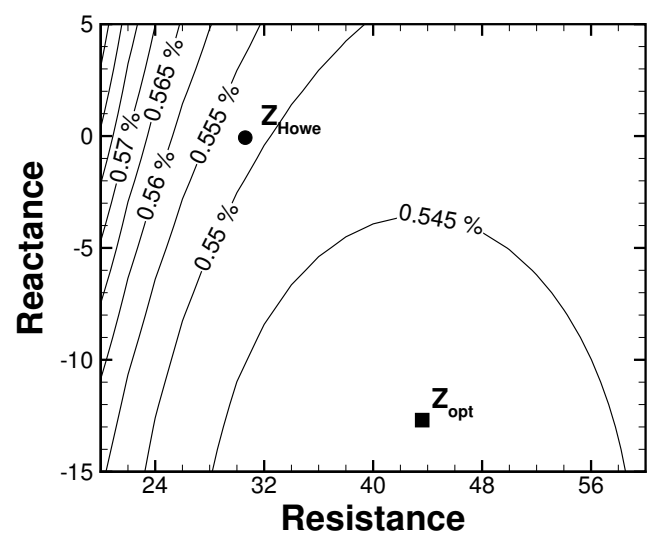

(b) Map in the impedance plane, when the source amplitude is fixed at its optimal value $C=-0.56+0.45 j$. The black square and circle show the position of the educed and modeled impedance, respectively.

Figure 10. Map of the reduced objective function at $500 \mathrm{~Hz}$, in the source amplitude (a) and impedance (b) planes. The exit impedance is fixed at its optimal value. Configuration with a $M_{b}=0.3$ bias flow.

the perforated plate with bias flow acts as a rigid wall.

An impedance eduction procedure developed for the nacelle inlets acoustic liners was applied to this set of experiments. The accuracy of the educed impedance values was carefully assessed. Without bias flow, the impedance of the 'perforated plate above cavity' system yields a significant acoustic attenuation along the duct, and therefore the eduction process works very well. The educed value is close to the one derived from models available in the literature, with low uncertainty on the results. On the contrary, with bias flow, the acoustic attenuation is very low, and the educed values exhibit very large resistance values. Consequently, the sensitivity of the objective function to the impedance is too low to obtain precise educed values. However, much more accurate results would be obtained if the perforated plate was studied in a different range of bias flow Mach number, in which the acoustic absorption would be higher.

\section{Acknowledgments}

Vincent Popie's PhD work is funded by the Direction Generale de l'Armement (DGA) which is gratefully acknowledged. The experimental part of this work was funded by the French National Research Agency (ANR) under grant no. ANR-08-SYSC-001, during the APAM project.

\section{References}

\footnotetext{
${ }^{1}$ Howe, M., "On the theory of unsteady high Reynolds number flow through a circular aperture," Proceedings of the Royal Society of London. A. Mathematical and Physical Sciences, Vol. 366, No. 1725, 1979, pp. 205-223.

${ }^{2}$ Zhou, L. and Boden, H., "The effect of combined high level acoustic excitation and bias flow on the acoustic properties of an in-duct orifice," 19th AIAA/CEAS Aeroacoustics conference, 2013, AIAA-2013-2128.

${ }^{3}$ Heuwinkel, C., Enghardt, L., Bake, F., Sadig, S., and Gerendas, M., "Establishment of a high quality database for the modelling of perforated liners," Proceedings of ASME Turbo Expo 2010, GT2010-22329.

${ }^{4}$ Hughes, I. and Dowling, A., "The absorption of sound by perforated linings," Journal of Fluid Mechanics, Vol. 218, No. 1, 1990, pp. 299-335.

${ }^{5}$ Primus, J., Piot, E., and Simon, F., "An adjoint-based method for liner impedance eduction: validation and numerical investigation," Journal of Sound and Vibration, Vol. 332, 2013, pp. 58-75.

${ }^{6}$ Primus, J., Piot, E., Simon, F., Jones, M., and Watson, W., "ONERA-NASA Cooperative Effort on Liner Impedance Eduction," 19th AIAA/CEAS Aeroacoustics conference, 2013, AIAA-2013-2273.

${ }^{7}$ Mendez, S. and Eldredge, J., "Acoustic modeling of perforated plates with bias flow for Large-Eddy Simulations," Journal of Computational Physics, Vol. 228, No. 13, 2009, pp. 4757-4772.

${ }^{8}$ Andreini, A., Facchini, B., Ferrari, L., Lenzi, G., Simonetti, F., and Peschiulli, A., "Experimental investigation on
} 
effusion liner geometries for aero-engine combustors: evaluation of global acoustic parameters," ASME Turbo Expo, 2012, GT2012-69853.

${ }^{9}$ Florenciano Merino, J., Etude de la reponse d'un ecoulement avec transfert parietal de masse a un forage acoustique: application au refroidissment des chambres de combustion aeronautiques, Ph.D. thesis, Universite de Pau et des pays de l'Adour, 2013.

${ }^{10}$ Scarpato, A., Tran, N., Ducruix, S., and Schuller, T., "Modeling the damping properties of perforated screens traversed by a bias flow and backed by a cavity at low Strouhal number," Journal of Sound and Vibration, Vol. 331, No. 2, 2012, pp. 276-290.

${ }^{11}$ Scarpato, A., Ducruix, S., and Schuller, T., "Optimal and off-design operations of acoustic dampers using perforated plates backed by a cavity," Journal of Sound and Vibration, Vol. 332, 2013, pp. 4856-4875.

${ }^{12}$ Cosic, B., Wassmer, D., Terhaar, S., and Paschereit, C., "Acoustic response of Helmholtz dampers in the presence of hot grazing flow," Journal of Sound and Vibration, Vol. 335, 2015, pp. 1-18.

${ }^{13}$ Andreini, A., Bianchini, C., Facchini, B., Vitale, I., and Turrini, F., "Numerical analysis of the acoustic and flow field associated with perforated liners with variable acoustic forcing," 19th AIAA/CEAS Aeroacoustics conference, 2013, AIAA2013-2156.

${ }^{14}$ Piot, E., Micheli, F., and Simon, F., "LDA investigation of the acoustic field above a perforated liner with grazing and bias flow," Inter-Noise 2009.

${ }^{15}$ Heuwinkel, C., Fischer, A., Rohle, I., Enghardt, L., Bake, F., Piot, E., and Micheli, F., "Characterization of a Perforated Liner by Acoustic and Optical Measurements," 16th AIAA/CEAS Aeroacoustics Conference, AIAA-2010-3765.

${ }^{16}$ Parisot-Dupuis, H., Simon, F., Piot, E., and Micheli, F., "Non-intrusive planar velocity-based nearfield acoustic holography in moving fluid medium," Vol. 133, No. 6, 2013, pp. 4087-4097.

${ }^{17}$ Tonon, D., Aeroacoustics of shear layers in internal flows: closed branches and wall perforations, Ph.D. thesis, Technische Universiteit Eindhoven, 2011.

${ }^{18}$ Canino, J. and Heister, S., "Contributions of orifice hydrodynamic instabilities to primary atomization," Atomization and Sprays, Vol. 19, No. 1, 2009, pp. 91-102.

${ }^{19}$ Lichtarowicz, A., Duggins, R., and Markland, E., "Discharge coefficients for incompressible non-cavitating flow through long orifices," J. Mech. Eng. Sci., Vol. 7, No. 2, 1965, pp. 210-219.

${ }^{20}$ Maa, D., "Potential of microperforated panel absorber," The Journal of the Acoustical Society of America, Vol. 104, No. 5, 1998, pp. 2861-2866.

${ }^{21}$ Crandall, I., Theory of Vibration System and Sound, Van Nostrand, 1926.

${ }^{22}$ Ingard, U., "On the theory and design of acoustic resonators," The Journal of the Acoustical Society of America, Vol. 25, No. 6, 1953, pp. 1037-1061.

${ }^{23}$ Melling, T., "The acoustic impendance of perforates at medium and high sound pressure levels," Journal of Sound and Vibration, Vol. 29, No. 1, 1973, pp. 1-65.

${ }^{24}$ Luong, T., Howe, M., and McGowan, R., "On the Rayleigh conductivity of a bias-flow aperture," Journal of fluids and structures, Vol. 21, No. 8, 2005, pp. 769-778.

${ }^{25}$ Jing, X. and Sun, X., "Effect of plate thickness on impedance of perforated plates with bias flow," AIAA journal, Vol. 38, No. 9, 2000, pp. 1573-1578.

${ }^{26}$ Tester, B., "The optimization of modal sound attenuation in ducts, in the absence of mean flow," Vol. 27, No. 4, 1973, pp. $477-513$.

${ }^{27}$ Tester, B., "The propagation and attenuation of sound in lined ducts containing uniform or "plug" flow," Vol. 28, No. 2, 1973, pp. 151-203.

${ }^{28}$ Gullaud, E., Mendez, S., Sensiau, C., Nicoud, F., and Poinsot, T., "Effect of multiperforated plates on the acoustic modes in combustors," Comptes Rendus Mecanique, Vol. 337, No. 6, 2009, pp. 406-414.

${ }^{29}$ Gullaud, E. and Nicoud, F., "Effect of perforated plates on the acoustics of annular combustors," AIAA Journal, Vol. 50, No. 12 , 2012, pp. 2629-2642. 